AperTO - Archivio Istituzionale Open Access dell'Università di Torino

\title{
On the first positive and negative excursion exceeding a given length
}

\section{This is a pre print version of the following article:}

Original Citation:

\section{Availability:}

This version is available http://hdl.handle.net/2318/1704016

since 2019-06-06T11:24:28Z

Published version:

DOI:10.1016/j.spl.2019.03.008

Terms of use:

Open Access

Anyone can freely access the full text of works made available as "Open Access". Works made available under a Creative Commons license can be used according to the terms and conditions of said license. Use of all other works requires consent of the right holder (author or publisher) if not exempted from copyright protection by the applicable law. 


\title{
On the first positive and negative excursion exceeding a given length
}

\author{
Roberta Sirovich $^{\mathrm{a}, *}$, Luisa Testa ${ }^{\mathrm{b}}$ \\ ${ }^{a}$ Department of Mathematics G. Peano, University of Torino, Via Carlo Alberto 10, 10123 \\ Torino, Italy \\ ${ }^{b}$ Department of Mathematics G. Peano, University of Torino, Via Carlo Alberto 10, 10123 \\ Torino, Italy
}

\begin{abstract}
For a one-dimensional diffusion process $X$, we derive the Laplace transform and the moments of the first time at which the age of an excursion above (or below) the level $x$ is longer than $u$. The result is then illustrated for diffusion processes that are found relevant in applications. In the context of pricing Parisian options, the Brownian motion and the Geometric Brownian motion are considered and the Laplace transform can be made explicit and explicit expression for the moments can be derived. In the context of neuronal modeling, the OrnsteinUhlenbeck process and the Cox-Ingersoll-Ross process are considered and the Laplace transform and the moments must be approximated by numerical inversion.
\end{abstract}

Keywords: diffusion processes, excursion theory, Parisian pricing

\section{Introduction}

Given a Markov process $X$ and a level $x$ that belongs to the state space, the purpose of excursion theory is to describe the evolution of $X$ in terms of the set $M=\left\{t: X_{t}=x\right\}$ and the pieces of the path that are defined over the complement of that $t$ set, the so called "excursions away from $x$ ".

Such a decomposition is particularly interesting in the regular and instantaneous case, where starting at $x$ the process is almost surely both visiting $x$ and away from $x$ during every arbitrarily small time interval $(0, t), t>0$. Then the random set $M$ is perfect and nowhere dense, see Bertoin (1996). This is the case of diffusion processes that, starting from $x$, almost surely have infinite crossings of the level $x$ in any arbitrarily small time interval.

In 1970 Itô showed how this case can be treated by viewing the excursions as "points" of a Poisson point process, indexed by local time and whose char-

\footnotetext{
${ }^{*}$ Corresponding author

Email addresses: roberta.sirovich@unito.it (Roberta Sirovich), luisa.testa@unito.it (Luisa Testa)
} 
acteristic measure describes many interesting features of the original process. Such remarkable results have been published in Itô (1972), that is considered the starting point for the excursion theory.

Later, Meyer discussed a more general case by introducing the entrance law, and Dynkin and Maisonneuve gave a complete analysis of excursions from a general set, see Meyer (1972), Dynkin (1968, 1971) and Maisonneuve (1975). Since then, a variety of applications have been developed, starting from the general theory of Maisonneuve, Getoor studied the excursion straddling a time $t$ and the first excursion exceeding $u$ in length, in the general setting of Hunt processes, Getoor (1979). For a complete treatment of excursion theory, readers can refer to Itô (1972), Rogers (1989), Pitman and Yor (1982) and Pitman and Yor (2003).

In this paper we focus on a particular excursion of diffusion processes. Specifically, let $X$ be a diffusion process with state space $I \in \mathbb{R}$. Given a fixed positive value $u$ and a given level $x \in I$, let us denote by $\left[G^{u,+}, D^{u,+}\right]$ the excursion interval that corresponds to the first excursion of $X$ above the level $x$, exceeding $u$ in length. On such interval, let us define the stopping time $H=G^{u,+}+u$, the first time the process remains constantly above the level $x$ for a time interval longer than $u$.

Starting from Getoor (1979) and Pitman and Yor (2003) we derive an analytical expression for the Laplace transform of the left endpoint of the excursion interval $G^{u,+}$, for any general diffusion process. Using the same approach, it is possible to derive the corresponding quantities for $G^{u,-}$, accounting for the first excursion of $X$ below the level $x$ exceeding $u$ in length. The results on $G^{u,+}$ can be directly transferred on $H$.

Our interest in $H$ comes from applications. The r. v. $H$ has been firstly introduced in Chesney et al. (1997) in order to study the so-called Parisian barrier options. The result presented in this paper solves the problem of Parisian pricing with underlying asset following any diffusion process, included the geometric Brownian motion. In the latter case, the method here presented provides a formula that requires a straightforward numerical inversion of a Laplace transform, see Section 4. Moreover, the r. v. $H$ has been recently introduced in Sirovich and Testa (2016) as the firing time in a single neuron Leaky Integrate and Fire model. The classical definition of firing time in this context is usually given as the first hitting time, of a given level, of the underlying membrane potential process. However, it has been proved Sirovich and Testa (2016) that such a 'hard threshold' hypothesis is rarely confirmed by real data from intracellular recordings. From there, the need of a 'soft threshold' definition has been addressed by introducing the r. v. $H$, so that the membrane potential process can cross the threshold level several times before firing. In order to check on data the predictive performances of the new firing model, theoretical results are here derived. Classical models for the underlying membrane potential process are the Orstein-Uhlenbeck and the Cox-Ingersoll-Ross processes. In such cases, the density of the r. v. $H$ can be approximated by a double numerical inversion of the Laplace transform, see Section 4.

The paper is organized as follows. Section 2 presents preliminaries on the 
basic relations between the distributions of hitting, occupation, and inverse local times. The main result of the paper is given in Section 3, where the Laplace transform $\mathbb{E}\left[\mathrm{e}^{-\lambda G^{u,+}}\right]$ is given for any general diffusion process. The result is then applied in Section 4 in order to calculate the Laplace transform of $H$ for three different diffusion processes: the Wiener process, the Ornstein-Uhlenbeck (OU) process and the Cox-Ingersoll-Ross (CIR) process. Section 5 contains numerical results of the Laplace inversions and the comparisons with Monte Carlo simulations.

\section{Preliminaries}

We will suppose through the article that $X$ is a diffusion process with state space some interval $I \subseteq \mathbb{R}$ and infinitesimal generator

$$
\mathcal{G}:=\frac{1}{2} a(x) \frac{d^{2}}{d x^{2}}+b(x) \frac{d}{d x},
$$

acting on a domain of functions subject to appropriate smoothness and boundary conditions. For all $x \in \operatorname{int}(I)$, the interior of $I, a(x)$ is assumed to be continuous and positive and $b(x)$ locally integrable. The process $X$ is a regular diffusion, i. e. for all $x \in \operatorname{int}(I)$ and $y \in I, \mathbb{P}^{x}\left(T_{y}<\infty\right)>0$, where $T_{y}=\inf \left\{t>0: X_{t}=y\right\}$.

For $x \in \operatorname{int}(I),\left(L_{t}^{x}\right)_{t \geq 0}$ is the local time process of $X$ at level $x$ and the occupation-measure formula says that, for any bounded measurable function $f$ supported in $\operatorname{int}(I)$,

$$
\int_{[0, t]} f\left(X_{s}\right) d\langle X, X\rangle_{s}=\int f(x) L_{t}^{x} d x
$$

where $\langle\cdot, \cdot\rangle$. is the quadratic variation process. It is well known that the map $t \rightarrow L_{t}^{x}$ is continuous and increasing with growth set $\left\{t \geq 0: X_{t}=x\right\}$.

Let $\left(\tau_{l}\right)_{l \geq 0}$ denote the right continuous inverse of the local time $L_{t}^{x}$,

$$
\tau_{l}=\inf \left\{s \geq 0: L_{s}^{x}>l\right\} .
$$

The process $\tau_{l}$ is a strictly increasing subordinator with

$$
\begin{aligned}
\mathbb{E}\left[\mathrm{e}^{-\lambda \tau_{l}}\right] & =\mathrm{e}^{-l \psi(\lambda)}, \\
\psi(\lambda) & =k+\gamma \lambda+\int_{] 0, \infty[}\left(1-\mathrm{e}^{-\lambda r}\right) \nu(d r),
\end{aligned}
$$

where $k=\psi(0) \geq 0$ is the killing rate, $\gamma \geq 0$ is the drift coefficient and $\nu$ is the Lévy measure on $] 0, \infty]$ satisfying $\int_{] 0, \infty[}(1 \wedge s) \nu(d s)<\infty$. As we are considering diffusion processes, any $x$ is of potential $\{0\}$ so that $\gamma=0$, see Getoor (1979).

It is well known, see Bertoin (1996), that either $x$ is recurrent, in which case $k=\psi(0)=0$ and $\mathbb{P}^{y}\left(\tau_{l}<\infty\right)=\mathbb{P}^{y}\left(L_{\infty}^{x}=\infty\right)=1$ for all $x, y \in \operatorname{int}(I)$, or $x$ 
is transient and $\tau_{l}$ has a jump to $\infty$ at an independent local time $L_{\infty}^{x}$ which is exponentially distributed with rate $k=\psi(0)>0$.

Using the same notation introduced in Getoor (1979), we denote by $h(u)=$ $\nu(] u, \infty])$ the tail of the Lévy measure $\nu$. Clearly, $h$ is a right continuous decreasing function on $] 0, \infty]$, with $h(\infty)=\nu(\{\infty\})=k$ and $\nu(d r)=-d h(r)$ on $(0, \infty)$. Moreover $\int_{] 0, t]} h(u) d u<\infty, \forall t<\infty$ and in particular $\lim _{u \rightarrow 0} u h(u)=0$ although $h\left(0^{+}\right)$is infinite.

In Pitman and Yor (2003) the authors proved the following result.

Theorem 1. The Laplace exponent $\psi(\lambda)$ of $\tau_{l}$ is given by

$$
\psi(\lambda)=\psi^{+}(\lambda)+\psi^{-}(\lambda)
$$

where for $\pm=+$ or -

$$
-\psi^{ \pm}(\lambda)= \pm \frac{1}{2} \frac{\Phi_{\lambda, \pm}^{\prime}(x)}{\Phi_{\lambda, \pm}(x)}= \pm\left.\frac{1}{2} \frac{d}{d z}\right|_{z=x \pm} \mathbb{P}^{z}\left[\exp \left(-\lambda T_{x}\right)\right]
$$

with $\Phi_{\lambda,+}$ and $\Phi_{\lambda,-}$ being respectively the decreasing and the increasing solutions $\Phi$ of $\mathcal{G} \Phi=\lambda \Phi$. Moreover,

$$
\psi^{ \pm}(\lambda)=\int_{] 0, \infty]}\left(1-e^{-\lambda r}\right) \nu^{ \pm}(d r),
$$

for some Lévy measure $\nu^{ \pm}$on $\left.] 0, \infty\right]$, with atoms at infinity of magnitudes

$$
\psi^{ \pm}(0)=- \pm \frac{1}{2} \lim _{\lambda \downarrow 0} \frac{\Phi_{\lambda, \pm}^{\prime}(x)}{\Phi_{\lambda, \pm}(x)} .
$$

Finally, the tail of the Lévy measure $\left.\left.h^{ \pm}(u)=\nu^{ \pm}(] u, \infty\right]\right)$ is given by

$$
h^{ \pm}(r)= \pm\left.\frac{1}{2} \frac{d}{d z}\right|_{z=x \pm} \mathbb{P}^{z}\left(T_{x} \geq r\right) \quad(0<r \leq \infty)
$$

and eq. 6an be rewritten as

$$
\psi^{ \pm}(\lambda)=\lambda \int_{] 0,+\infty]} e^{-\lambda r} h^{ \pm}(r) d r .
$$

Let the occupation times $A_{t}^{ \pm}$be defined as

$$
A_{t}^{+}=\int_{[0, t]} \mathbf{1}\left(X_{s}>x\right) d s, \quad A_{t}^{-}=\int_{[0, t]} \mathbf{1}\left(X_{s} \leq x\right) d s .
$$

As $t=A_{t}^{+}+A_{t}^{-}$, we have the decomposition $\tau_{l}=A_{\tau_{l}}^{+}+A_{\tau_{l}}^{-}$. According to (Pitman and Yor, 2003, Corollary 2), if $X$ is a recurrent process, the processes $A_{\tau_{l}}^{+}$and $A_{\tau_{l}}^{-}$are two independent subordinators with Laplace exponents

$$
\mathbb{E}^{x}\left[\exp \left(-\lambda A_{\tau_{l}}^{ \pm}\right)\right]=\exp \left[-l \psi^{ \pm}(\lambda)\right]
$$


and hence the subordinator $\tau_{l}$ is the sum of two independent subordinators. In the transient case, the two processes $A_{\tau_{l}}^{ \pm}$are independent subordinators only conditionally on $L_{\infty}^{x}$. Otherwise, independence fails and only one of them is a subordinator with a jump to $\infty$. The other one indeed is constant for every time larger than $\tau_{L_{\infty}^{x}}$.

The above results can be interpreted in the framework of Itô's excursion theory. Let $\bar{M}$ denote the closure of $\left\{t \geq 0: X_{t}(\omega)=x\right\}$. The complement of $\bar{M}$ in $\mathbb{R}^{+}$is expressed, in the canonical form, as a disjoint countable union of open intervals $\bigcup_{j}\left(a_{j}, b_{j}\right)$. The paths defined by $X_{a_{j}+s}$, for $0 \leq s<b_{j}-a_{j}$, are the so called excursions of $X$ away from $x$. The length of the excursion interval $\left(b_{j}-a_{j}\right)$, is called lifetime of the excursion. As the excursion intervals are precisely the open intervals of the type $\left(\tau_{l^{-}}, \tau_{l}\right)$ for $\tau_{l^{-}}<\tau_{l}$, this suggested the use of the inverse local time as a new time scale to work on the excursions of $X$. More formally, let us introduce the excursion process $\Pi$ that takes values in the excursion space

$$
U=\left\{f: \mathbb{R}^{+} \rightarrow \mathbb{R} \text { continuous : } f^{-1}(\mathbb{R}-\{x\})=(0, \zeta) \text { for some } \zeta>0\right\} .
$$

The process $\Pi$ has a point $e_{l}$ at time $l$ if and only if $X$ makes an excursion at local time $l$ and

$$
e_{l}(s)=X_{\tau_{l^{-}}+s}, \quad 0 \leq s<\tau_{l}-\tau_{l^{-}} .
$$

The excursion process has been proved to be a Poisson Point Process stopped at $L_{\infty}^{x}$ with intensity $n$, where $n$ is a $\sigma$-finite measure on the space of excursions $U$ called the excursion measure, see Rogers (1989). As the lengths of the excursions correspond to the amplitude of the jumps of the process $\tau_{l}$, we have that

$$
n(\zeta \in d t)=\nu(d t)
$$

where $\nu$ is the Lévy measure of the subordinator $\tau_{l}$ with characteristic exponent given in 3 .

By continuity of the trajectories, each excursion away from $x$ is either a + excursion which lies entirely in $[x, \infty)$, or a - excursion which lies entirely in $(-\infty, x]$. Following the same arguments, the lengths of the + excursions correspond to the amplitudes of the jumps of the process $A_{\tau_{l}}^{+}$and

$$
n(+ \text { excursions, } \zeta \in d t)=\nu^{+}(d t) \text {. }
$$

Hence, the study of the distribution of the excursions' lifetimes is strictly connected to the study of the inverse local time.

\section{Results}

Let $G^{u}$ be the left endpoint of the first excursion exceeding $u>0$ in length and let $D^{u}$ be its right endpoint. In Getoor (1979) the Laplace transform of $G^{u}$ has been derived. We are interested in separating the excursions above the level $x$ and below the level $x$. Hence, let $G^{u,+}$ and $D^{u,+}$ be respectively the left and right endpoints of the first excursion above the level $x$ and exceeding $u$ in length. Our main result is the Laplace transform of $G^{u,+}$. 
Theorem 2. Let $X$ be a recurrent or transient process with $\psi^{+}(0)=\psi(0)$ (or equivalently $\left.\psi^{-}(0)=0\right)$, then

$$
\mathbb{E}^{x}\left[e^{-\lambda G^{u,+}}\right]=\frac{h^{+}(u)}{h^{+}(u)+\int_{] 0, u]}\left(1-e^{-\lambda r}\right) \nu(d r)+\int_{] u, \infty]}\left(1-e^{-\lambda r}\right) \nu^{-}(d r)},
$$

where $\nu, h^{+}$and $\nu^{-}$have been defined in eqs. (3), (8) and (6) respectively.

Proof. Let us remark that condition $\psi^{+}(0)=\psi(0)$ ensures that the last excursion with infinite length is above the level $x$ a. s., see Pitman and Yor (2003).

Let $\tau_{l}$ be the right continuous inverse of the local time $L_{t}^{x}$. We write $\tau_{l}=\tau_{l}^{1}+\tau_{l}^{2}$, where (conditionally on $\left.l<L_{\infty}^{x}\right) \tau_{l}^{1}$ and $\tau_{l}^{2}$ are two independent subordinators with exponents $\int_{] 0, u]}\left(1-e^{-\lambda r}\right) \nu(d r)$ and $\int_{] u,+\infty]}\left(1-e^{-\lambda r}\right) \nu(d r)$ respectively. Hence, $\tau_{l}^{1}$ contains all the jumps of $\tau$ which are less than or equal to $u$, while $\tau_{l}^{2}$ contains all the jumps of $\tau$ which exceed $u$.

Moreover, we write $\tau_{l}^{2}=\tau_{l}^{2,+}+\tau_{l}^{2,-}$, where (conditionally on $l<L_{\infty}^{x}$ ) $\tau_{l}^{2, \pm}$ are two independent subordinators accounting for the + excursions and the - excursions. Hence they have exponents $\int_{] u,+\infty]}\left(1-e^{-\lambda r}\right) \nu^{+}(d r)$ and $\int_{] u,+\infty]}\left(1-e^{-\lambda r}\right) \nu^{-}(d r)$ respectively.

Let $R$ be the (local) time of the first jump of $\tau_{l}^{2,+}$. Then $R$ is the (local) time of the first jump of $\tau_{l}$ that exceeds $u$ and that corresponds to a + excursion. As the time of the first event in the excursion process, $R$ is exponentially distributed with parameter $h^{+}(u)$. Moreover, $R=L_{G^{u,+}}^{x}$ and $G^{u,+}=\tau_{R^{-}}^{1}+\tau_{R^{-}}^{2,+}+\tau_{R^{-}}^{2,-}=$ $\tau_{R^{-}}^{1}+\tau_{R^{-}}^{2,-}$ as $\tau_{R^{-}}^{2,+}=0$.

Considering that $R$ is independent from $\tau^{1}$ and $\tau^{2,-}$, it follows

$$
\begin{aligned}
\mathbb{P}^{x}\left(G^{u,+} \leq v\right) & =\mathbb{P}^{x}\left(\tau_{R^{-}}^{1}+\tau_{R^{-}}^{2,-} \leq v\right) \\
& =\int_{0}^{\infty} \mathbb{P}^{x}\left(\tau_{l^{-}}^{1}+\tau_{l^{-}}^{2,-} \leq v \mid R=l\right) \mathbb{P}(R \in d l) \\
& =\int_{0}^{\infty} \mathbb{P}^{x}\left(\tau_{l^{-}}^{1}+\tau_{l^{-}}^{2,-} \leq v\right) h^{+}(u) e^{-l h^{+}(u)} d l \\
& =\int_{0}^{\infty} \mathbb{P}^{x}\left(\tau_{l}^{1}+\tau_{l}^{2,-} \leq v\right) h^{+}(u) e^{-l h^{+}(u)} d l,
\end{aligned}
$$

where the last equality holds true as $l \rightarrow \tau_{l}^{1}$ and $l \rightarrow \tau_{l}^{2,-}$ have at most a countable number of discontinuities.

Finally, taking Laplace transforms and expressing the expectations as functions of the tail probabilities, we obtain

$$
\begin{aligned}
\mathbb{E}^{x}\left[\mathrm{e}^{-\lambda G^{u,+}}\right] & =\int_{0}^{\infty} \mathbb{E}^{x}\left[e^{-\lambda\left(\tau_{l}^{1}+\tau_{l}^{2,-}\right)}\right] h^{+}(u) e^{-l h^{+}(u)} d l \\
& =\int_{0}^{\infty} \mathbb{E}^{x}\left[e^{-\lambda \tau_{l}^{1}}\right] \mathbb{E}^{x}\left[e^{-\lambda \tau_{l}^{2,-}}\right] h^{+}(u) e^{-l h^{+}(u)} d l
\end{aligned}
$$


Considering that $\tau_{l}^{1}$ and $\tau_{l}^{2,-}$ have exponents $\int_{] 0, u]}\left(1-e^{-\lambda r}\right) \nu(d r)$ and $\int_{] u,+\infty]}(1-$ $\left.e^{-\lambda r}\right) \nu^{-}(d r)$ respectively, we get

$$
\begin{aligned}
\mathbb{E}^{x}\left[\mathrm{e}^{-\lambda G^{u,+}}\right] & =\int_{0}^{\infty} h^{+}(u) e^{-l\left[h^{+}(u)+\int_{j 0, u]}\left(1-e^{-\lambda r}\right) \nu(d r)+\int_{] u,+\infty]}\left(1-e^{-\lambda r}\right) \nu^{-}(d r)\right]} d l \\
& =\frac{h^{+}(u)}{h^{+}(u)+\int_{] 0, u]}\left(1-e^{-\lambda r}\right) \nu(d r)+\int_{] u,+\infty]}\left(1-e^{-\lambda r}\right) \nu^{-}(d r)}
\end{aligned}
$$

Corollary 3. Eq. 14 can be rewritten as

$$
\mathbb{E}^{x}\left[e^{-\lambda G^{u,+}}\right]=\frac{h^{+}(u)}{\int_{] 0, u]} \lambda e^{-\lambda r} h^{+}(r) d r+e^{-\lambda u} h^{+}(u)+\psi^{-}(\lambda)} .
$$

Remark: As we will see in Section 4 , eq. (15) turns out to be the most convenient expression for the numerical calculation of the Laplace transform of $G^{u,+}$.

Remark: Under the hypotheses of Theorem 2, from eq. 15 it is easy to verify that $\mathbb{P}^{x}\left(G^{u,+}<\infty\right)=1$.

From the Laplace transform, a recursive formula for any $k$-th moment of the r. v. $G^{u,+}$ is deduced in the following theorem.

Theorem 4. Let $X$ be recurrent or transient with $\psi^{+}(0)=\psi(0)$ (or equivalently $\left.\psi^{-}(0)=0\right)$. The $k-$ th moment of $G^{u,+}$ can be recursively calculated as

$$
\mathbb{E}^{x}\left[\left(G^{u,+}\right)^{k}\right]=\left.(-1)^{k+1} \sum_{j=0}^{k-1}\left(\begin{array}{c}
k \\
j
\end{array}\right) \frac{d^{j}}{d \lambda^{j}} \frac{1}{D(\lambda)} \frac{d^{k-j}}{d \lambda^{k-j}} D(\lambda)\right|_{\lambda=0},
$$

where $D(\lambda)$ is

$$
D(\lambda)=\int_{] 0, u]} \lambda e^{-\lambda r} h^{+}(r) d r+e^{-\lambda u} h^{+}(u)+\psi^{-}(\lambda),
$$

with higher order derivatives in $\lambda=0$

$$
\left.\frac{d^{n}}{d \lambda^{n}} D(\lambda)\right|_{\lambda=0}=\int_{] 0, u]}(-1)^{n+1} n r^{n-1} h^{+}(r) d r+(-u)^{n} h^{+}(u)+\left.\frac{d^{n}}{d \lambda^{n}} \psi^{-}(\lambda)\right|_{\lambda=0} .
$$

Proof. Rewriting eq. 15) as $\mathbb{E}^{x}\left[e^{-\lambda G^{u,+}}\right]=h^{+}(u) / D(\lambda)$ and recalling that

$$
\mathbb{E}^{x}\left[\left(G^{u,+}\right)^{k}\right]=\left.(-1)^{k} \frac{d^{k}}{d \lambda^{k}} \mathbb{E}^{x}\left[\mathrm{e}^{-\lambda G^{u,+}}\right]\right|_{\lambda=0},
$$

we get

$$
\mathbb{E}^{x}\left[\left(G^{u,+}\right)^{k}\right]=\left.(-1)^{k} h^{+}(u) \frac{d^{k}}{d \lambda^{k}} \frac{1}{D(\lambda)}\right|_{\lambda=0} .
$$

Finally, by the quotient formula given in Slevinsky and Safouhi (2009), we get the convenient expression (16). 
Corollary 5. The expected value of $G^{u,+}$ is

$$
\mathbb{E}^{x}\left[G^{u,+}\right]=\frac{\int_{] 0, u]} h^{+}(r) d r+\left.\frac{d}{d \lambda} \psi^{-}(\lambda)\right|_{\lambda=0}}{h^{+}(u)}-u
$$

\section{Examples}

In the following Sections we derive an expression for eq. (18), as explicit as possible, when $X$ is a Wiener process, an OU process and a CIR process. Let us recall that

$$
\begin{aligned}
\mathbb{E}^{X_{0}}\left[\mathrm{e}^{-\lambda H}\right] & =\mathrm{e}^{-\lambda u} \mathbb{E}^{X_{0}}\left[\mathrm{e}^{-\lambda G^{u,+}}\right] \\
& =\mathrm{e}^{-\lambda u} \mathbb{E}^{X_{0}}\left[\mathrm{e}^{-\lambda T_{x}}\right] \mathbb{E}^{x}\left[\mathrm{e}^{-\lambda G^{u,+}}\right]
\end{aligned}
$$

where $\mathbb{E}^{x}\left[\mathrm{e}^{-\lambda G^{u,+}}\right]$ is given by eq. 15 .

\subsection{Brownian Motion}

In the Wiener case the Laplace transform of the r. v. $H$ can be derived explicitly and the same expression given in Chesney et al. (1997) and Sirovich and Testa (2016) is retrieved.

Proposition 1. Let us consider the Wiener process with non negative drift which satisfies the equation:

$$
d X_{t}=\mu d t+\sigma d W_{t}, \quad \mu \geq 0 \quad X_{0}=0 .
$$

Then the Laplace transform of the r. $v . H$ is given by

$$
\mathbb{E}^{0}\left(e^{-\lambda H}\right)=\exp \left[\frac{\mu x}{\sigma^{2}}-\frac{x}{\sigma} \sqrt{2\left(\lambda+\frac{|\mu|^{2}}{2 \sigma^{2}}\right)}\right] \frac{\phi\left(\frac{\mu}{\sigma} \sqrt{u}\right)}{\phi\left(\sqrt{2\left(\lambda+\frac{|\mu|^{2}}{2 \sigma^{2}}\right) u}\right)},
$$

and

$$
\mathbb{E}^{0}[H]=\left(\frac{x}{\mu}+u\right)+\frac{\sigma^{2}}{\mu^{2}}\left(1-\frac{1}{\phi\left(\frac{\mu}{\sigma} \sqrt{u}\right)}\right),
$$

where

$$
\phi(z)=\int_{0}^{+\infty} r e^{\left(z r-\frac{r^{2}}{2}\right)} d r=1+\sqrt{\frac{\pi}{2}} z e^{\frac{z^{2}}{2}}\left[1+\operatorname{erf}\left(\frac{z}{\sqrt{2}}\right)\right] .
$$

Proof. Under the assumption $\mu \geq 0$, we have $\psi^{+}(0)=0$ so, the Laplace transform of $H$ can be calculated by eq. $[18)$, where $\mathbb{E}^{x}\left[e^{-\lambda G^{u,+}}\right]$ is given by eq. [15]. Let us first calculate $\psi^{ \pm}(\lambda)$. It is well know that, Siegert (1951),

$$
\mathbb{E}^{y}\left[\mathrm{e}^{-\lambda T_{x}}\right]=\mathrm{e}^{-\left(\sqrt{\mu^{2}+2 \lambda \sigma^{2}} \frac{|y-x|}{\sigma^{2}}+\mu \frac{(y-x)}{\sigma^{2}}\right)} .
$$


Using eq. (5), $\psi^{ \pm}(\lambda)$ takes the following expression

$$
\psi^{ \pm}(\lambda)=\frac{1}{2 \sigma^{2}}\left(\sqrt{2 \lambda \sigma^{2}+\mu^{2}} \pm \mu\right) .
$$

An explicit equation for $h^{ \pm}(u)$ can be obtained from eq. (9) by inverting $\psi^{+}(\lambda) / \lambda$. We get

$$
h^{ \pm}(u)=\frac{1}{2 \sigma^{2}}\left[\mu \operatorname{erf}\left(\frac{\mu \sqrt{u}}{\sqrt{2} \sigma}\right) \pm \mu+\frac{\sqrt{2} \sigma}{\sqrt{\pi u}} \exp \left(-\frac{\mu^{2} u}{2 \sigma^{2}}\right)\right] .
$$

By replacing eq. (23) and eq. (24) in eq. (15) we get the desired result.

The expected value of $H$ is a straightforward calculation of the derivative of the Laplace transform 20 at $\lambda=0$.

\subsection{The Geometric Brownian Motion}

The r. v. $H$ has been studied in Chesney et al. (1997) where the so called Parisian barrier options have been introduced. They are path dependent options. For example, the owner of a Parisian down-and-out call loses the option if the value of the underlying asset price reaches the knock-out level and remains long enough under this level. For pricing Parisian options the problem is reduced to finding the Laplace transform of the Parisian stopping time, which is the r. v. here denoted as $H$. In Chesney et al. (1997) and Dassios and Wu (2010) two different methods are presented to find an explicit form for the Laplace transform of $H$, when the underlying asset follows a Geometric Brownian motion. The two methods cannot be generalised to different processes, as they heavily rely on properties of the Brownian motion. The result here presented is an alternative method to derive an explicit Laplace transform of the stopping time which turns out to be easily invertible. Moreover, the result here presented is general, it holds for any one dimensional diffusion process and hence can be applied for pricing Parisian option when the underlying asset price is another diffusion process.

Let $Q$ denote the risk neutral probability. Under $Q$, the dynamics of the underlying asset follow a geometric Brownian motion $S$ given by

$$
d S_{t}=S_{t}\left([r-\delta] d t+\gamma d W_{t}\right), \quad S_{0}=s_{0},
$$

where $W$ is a standard Brownian motion under $Q$, and $r, s_{0}$ and $\gamma$ are positive constants. By Itô formula, it follows that the solution is a transformation of the standard Brownian motion $W$ given by

$$
S_{t}=s_{0} \exp \left(\left[r-\delta-\gamma^{2} / 2\right] t+\gamma W_{t}\right) .
$$

Hence, the r. v. $H$ for the process $S$ and a given level $b$ can be obtained as the r. v. $H$ for the Brownian motion with drift and diffusion parameters given by

$$
\begin{aligned}
& \mu=r-\delta-\gamma^{2} / 2 \\
& \sigma=\gamma
\end{aligned}
$$


and level $x=\log \left(b / s_{0}\right)$. So, for underlying asset following a Geometric Brownian motion, the problem of pricing Parisian can be reduced to finding the Laplace transform of the r. v. $H$ for a Brownian motion with suitable drift and diffusion coefficients and tranformed level $x$. Eqs. 20 and (21) can be trivially rewritten and will give the explicit expression for the Laplace transform and the first moment of the Parisian stopping time.

Remark 1. The two-sided Parisian options have been introduced in Dassios and Wu (2010). The fully symmetric case has been studied in Dassios and Lim (2017). Let us remark that in this latter case the Laplace transform of the two-sided Parisian time is given in Getoor (1979), Proposition (9.2).

\subsection{Ornstein-Uhlenbeck process}

Unfortunately, for the OU process the only closed form result is the expression for $\psi^{ \pm}(\lambda)$, which cannot be inverted analytically. Hence, in order to recover $h^{ \pm}(u)$ we should rely on numerical algorithms that will be presented in Section 5 and we will only have numerical approximations of $\mathbb{E}^{x_{0}}\left[\mathrm{e}^{-\lambda H}\right]$ and of $\mathbb{E}^{x_{0}}[H]$.

Proposition 2. Let us consider an OU process given by

$$
d X_{t}=\left(-\beta X_{t}+\mu\right) d t+\sigma d W_{t}, \quad X_{0}=x_{0}
$$

where $\beta>0, \mu \geq 0$ and $\sigma>0$. We have that

$$
\psi^{ \pm}(\lambda)=\left(\frac{\lambda}{\sqrt{\beta}}\right) \frac{H_{-\lambda / \beta-1}\left( \pm \frac{x \sqrt{\beta}}{\sigma} \mp \frac{\mu}{\sigma \sqrt{\beta}}\right)}{H_{-\lambda / \beta}\left( \pm \frac{x \sqrt{\beta}}{\sigma} \mp \frac{\mu}{\sigma \sqrt{\beta}}\right)},
$$

where $\psi^{ \pm}$is given by (5) and $H_{v}(z)$ denotes the Hermite function of degree $v$, see Lebedev (1972).

Proof. The result follows from eq. (5) and from the given expressions for the Laplace transform of the first passage time $T_{x}$

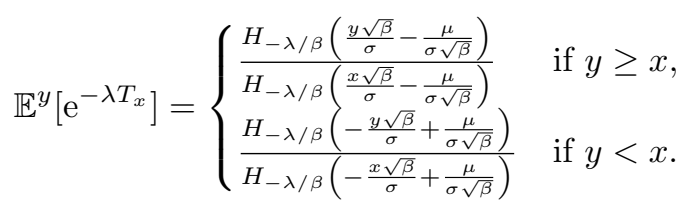

\subsection{Cox-Ingersoll-Ross process}

As in the previous case, also for the CIR process, the only closed form result is the expression for $\psi^{ \pm}(\lambda)$. In Section 5 we will recover a numerical approximation of $\mathbb{E}^{x_{0}}\left[\mathrm{e}^{-\lambda H}\right]$ and $\mathbb{E}^{x_{0}}[H]$. 
Proposition 3. Let us consider a CIR process, solution of the equation

$$
d X_{t}=\left(-\gamma X_{t}+\mu\right) d t+\sigma \sqrt{X_{t}} d W_{t}, \quad X_{0}=x_{0}>0,
$$

where $\gamma>0, \mu \geq 0, \sigma>0$, and $2 \mu \geq \sigma^{2}$. Then,

$$
\psi^{+}(\lambda)=\frac{\lambda}{\sigma^{2}} \frac{\Psi\left(\frac{\lambda}{\gamma}+1 ; \frac{2 \mu}{\sigma^{2}}+1 ; \frac{2 x \gamma}{\sigma^{2}}\right)}{\Psi\left(\frac{\lambda}{\gamma} ; \frac{2 \mu}{\sigma^{2}} ; \frac{2 x \gamma}{\sigma^{2}}\right)},
$$

and

$$
\psi^{-}(\lambda)=\frac{\lambda}{2 \mu} \frac{\Phi\left(\frac{\lambda}{\gamma}+1 ; \frac{2 \mu}{\sigma^{2}}+1 ; \frac{2 x \gamma}{\sigma^{2}}\right)}{\Phi\left(\frac{\lambda}{\gamma} ; \frac{2 \mu}{\sigma^{2}} ; \frac{2 x \gamma}{\sigma^{2}}\right)},
$$

where $\Psi$ denote the Kummer function of the second kind and $\Phi$ denote the Kummer function of the first kind, see Olver et al. (2010).

Proof. We assume that $2 \mu \geq \sigma^{2}$ as under this condition the origin is inaccessible and the process $X$ ergodic. The expression for $\psi^{ \pm}(\lambda)$ is recovered from eq. (5) and the fact that, for $y>x$

$$
\mathbb{E}^{y}\left[e^{-\lambda T_{x}}\right]=\frac{\Psi\left(\frac{\lambda}{\gamma} ; \frac{2 \mu}{\sigma^{2}} ; \frac{2 y \gamma}{\sigma^{2}}\right)}{\Psi\left(\frac{\lambda}{\gamma} ; \frac{2 \mu}{\sigma^{2}} ; \frac{2 x \gamma}{\sigma^{2}}\right)},
$$

and, for $y<x$

$$
\mathbb{E}^{y}\left[e^{-\lambda T_{x}}\right]=\frac{\Phi\left(\frac{\lambda}{\gamma} ; \frac{2 \mu}{\sigma^{2}} ; \frac{2 y \gamma}{\sigma^{2}}\right)}{\Phi\left(\frac{\lambda}{\gamma} ; \frac{2 \mu}{\sigma^{2}} ; \frac{2 x \gamma}{\sigma^{2}}\right)} .
$$

\section{Numerical Results}

In this Section we illustrate the numerical details necessary to deduce an approximation of the probability density function of the r. v. $H$ and its expectation for the three processes proposed in Section 4. The goal is twofold. Firstly, we show that the main result of the paper, illustrated in Theorem 2, can be successfully used to derive the desired quantities. Secondly, the comparison between numerical outputs and simulated quantities confirms the validity of our theoretical results.

For each of the three examples we calculate numerically an approximation of the density of the r. v. $H$ and plot it against the histogram of sample values of the r. v. $H$, obtained from $N=10000$ Monte Carlo simulated trajectories of the underlying diffusion process. As the transition density functions are known for each of the three processes, the trajectories are simulated exactly. Numerical inversions of the Laplace transforms have been implemented in Wolfram Mathematica using the Gaver-Stehfest algorithm, see Stehfest (1970). Moreover, we plot the numerical approximation obtained for the expected value $\mathbb{E}(H)$ against 

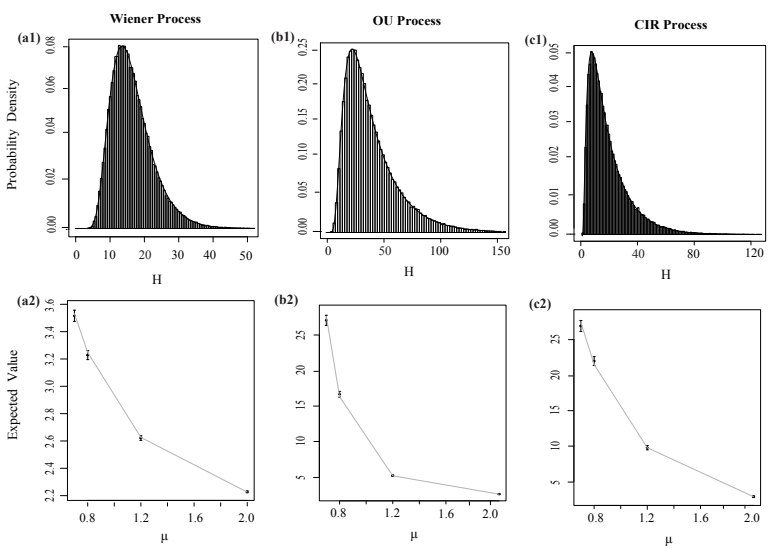

Figure 1: First row: simulated (bar plot) and numerical (continuous line) densities. Second row: numerical expected values (grey solid line) and simulated $95 \%$ confidence intervals (black vertical bars). Wiener process: $x=10, x_{0}=0, u=2, \sigma=1, \mu=0.7$. OU process: $x=10, x_{0}=0, u=2, \sigma=1, \mu=0.8, \beta=0.08$. CIR process: $x=10, x_{0}=2.5, u=2$, $\sigma=1, \mu=1.2, \gamma=0.08$.

the confidence intervals for the sample mean obtained from Monte Carlo simulations.

The Wiener process is the only case in which an explicit expression for the Laplace transform of $H$ can be derived. Hence, the approximated density of $H$ can be easily obtained by the numerical inversion of eq. 20). Fig. 1 (panel a1) shows the complete agreement between the numerical and the simulated densities of $H$. Fig. 1 (panel a2) plots numerical and empirical expected values of $H$.

As previously mentioned, for both the OU and the CIR processes, $\mathbb{E}^{X_{0}}\left[e^{-\lambda H}\right]$ cannot be derived in closed expression. Hence we need to perform two Laplace inversions to obtain the density of $H$. Firstly, we invert $\psi^{ \pm}(\lambda) / \lambda$ to get a numerical expression for $h^{ \pm}$and secondly we invert eq. 115). The obtained results are shown in Fig. 1. In both cases the numerical results are completely satisfactory and simulated data fit perfectly.

Bertoin, J., 1996. Lévy processes. volume 121 of Cambridge Tracts in Mathematics. Cambridge University Press, Cambridge.

Chesney, M., Jeanblanc-Picqué, M., Yor, M., 1997. Brownian excursions and Parisian barrier options. Adv. in Appl. Probab. 29, 165-184.

Dassios, A., Lim, J.W., 2017. An analytical solution for the two-sided parisian stopping time, its asymptotics, and the pricing of parisian options. Mathematical Finance 27, 604-620.

Dassios, A., Wu, S., 2010. Perturbed brownian motion and its application to parisian option pricing. Finance and Stochastics 14, 473-494. 
Dynkin, E.B., 1968. On extensions of a markov process. Theory Probab. Appl. $13,672-676$.

Dynkin, E.B., 1971. Wanderings of a markov process. Theory Probab. Appl. $16,401-428$.

Getoor, R.K., 1979. Excursions of a Markov process. Ann. Probab. 7, 244-266.

Itô, K., 1972. Poisson point processes attached to Markov processes, in: Proceedings of the Sixth Berkeley Symposium on Mathematical Statistics and Probability (Univ. California, Berkeley, Calif., 1970/1971), Vol. III: Probability theory, Univ. California Press, Berkeley, Calif.. pp. 225-239.

Lebedev, N.N.a., 1972. Special functions and their applications. Dover Publications, Inc., New York. Revised edition, translated from the Russian and edited by Richard A. Silverman, Unabridged and corrected republication.

Maisonneuve, B., 1975. Exit systems. Ann. Probability 3, 399-411.

Meyer, P.A.a., 1972. Processus de Poisson ponctuels, d'après K. Itô", in: Séminaire de Probabilités, VI (Univ. Strasbourg, année universitaire 19701971). Springer, Berlin, pp. p. 253. Lecture Notes in Math., Vol. 258.

Olver, F.W.J., Lozier, D.W., Boisvert, R.F., Clark, C.W. (Eds.), 2010. NIST handbook of mathematical functions. U.S. Department of Commerce, National Institute of Standards and Technology, Washington, DC; Cambridge University Press, Cambridge.

Pitman, J., Yor, M., 1982. A decomposition of Bessel bridges. Z. Wahrsch. Verw. Gebiete 59, 425-457.

Pitman, J., Yor, M., 2003. Hitting, occupation and inverse local times of onedimensional diffusions: martingale and excursion approaches. Bernoulli 9, $1-24$.

Rogers, L.C.G., 1989. A guided tour through excursions. Bull. London Math. Soc. 21, 305-341.

Siegert, A.J., 1951. On the first passage time probability problem. Physical Review 81, 617.

Sirovich, R., Testa, L., 2016. A new firing paradigm for integrate and fire stochastic neuronal models. Math. Biosci. Eng. 13, 597-611.

Slevinsky, R.M., Safouhi, H., 2009. New formulae for higher order derivatives and applications. J. Comput. Appl. Math. 233, 405-419.

Stehfest, H., 1970. Algorithm 368: Numerical inversion of laplace transforms. Communications of the ACM 13, 47-49. 